\title{
DiFS: Distributed Flow Scheduling for Adaptive Switching in FatTree Data Center Networks
}

\author{
Wenzhi Cui ${ }^{1}$, Ye $\mathrm{Yu}^{2}$, Chen Qian ${ }^{3, *}$
}

\begin{abstract}
Data center networks leverage multiple parallel paths connecting end host pairs to offer high bisection bandwidth for cluster computing applications. However, the state-of-the-art routing protocols such as Equal Cost Multipath (ECMP) is load-oblivious due to the static flow-to-link assignment. They may cause bandwidth loss due to flow collisions. Recently proposed centralized scheduling algorithm or host based adaptive routing that requires network-wide state information may suffer from scalability problems. In this paper, we present Distributed Flow Scheduling (DiFS), a new adaptive switching method, for FatTree data center networks, which is a localized and switch-only solution. DiFS allows switches to cooperate to avoid over-utilized links and find available paths without centralized control. DiFS is scalable and can react quickly to dynamic traffic because it is independently executed on switches and requires no synchronization. DiFS provides global bounds of flow balance based on local optimization. Extensive simulations show that the aggregate throughput of DiFS using various traffic patterns is much better than that of ECMP, and is similar to or higher than those of two representative protocols that use network-wide optimization.
\end{abstract}

Keywords: Data center networks, adaptive switching, flow scheduling

\section{Introduction}

The growing importance of cloud-based applications and big data processing has led to the deployment of large-scale data center networks that carry a tremendous amount of traffic. Recently proposed data center network architectures primarily focus on using commodity Ethernet switches to build hierarchical trees. These data center networks usually use FatTree or similar topologies [1] [2] [3] [4] [5]. These topologies provide multiple equal-cost paths between any pair of end hosts and hence significantly increase bisection bandwidth. To fully utilize the path diversity, an ideal switching scheme should allow flows to avoid overutilized links and take alternative paths, called adaptive switching. In this work, we focus on the investigation of throughput improvement by switching methods. Other approaches such as application layer virtual ma-

\footnotetext{
${ }^{*}$ Corresponding author

${ }^{1}$ Department of Computer Science, the University of Texas at Austin, TX, 78712, E-mail: wc8348@cs.utexas.edu

${ }^{2}$ Department of Computer Science, University of Kentucky, KY, 40506, E-mail: ye.yu@uky.edu

${ }^{3}$ Department of Computer Science, University of Kentucky, KY, 40506, E-mail: qian@cs.uky.edu
}

Preprint submitted to Computer Networks chine placement and transport layer protocols are out of the scope of this paper.

Most state-of-the-art data center networks rely on layer-3 Equal Cost Multipath (ECMP) protocol [6] to assign flows to available links using static flow hashing. Being simple and efficient. However, ECMP is loadoblivious, because the flow-to-path assignment does not account current network utilization. As a result, ECMP may cause flow collisions on particular links and create hot spots.

We classify the recently proposed methods of improving the bandwidth utilization in data center networks into three categories: centralized, host-based, and switch-only.

- Centralized solutions utilize the recent advances in Software Defined Networking (SDN), which allows a central controller to perform control plane tasks and install forwarding entries to switches via a special protocol such as OpenFlow [7]. A typical centralized solution Hedera [8] relies on a central controller to find a path for each flow or assign a single core switch to deal with all flows to each destination host. Centralized solutions may face scalability problems [9], because traffic in today's data center networks requires parallel and fast path selection according to recent measurement studies 
$[10,11]$. A recent work Fastpass [12] demonstrates very promising performance. Fastpass uses an arbiter to control the time at which each packet should be transmitted and the path to use for the packet.

- Host-based methods, such as DARD [3], can be run without a central control. These methods enable end systems to monitor the network bandwidth utilization and then select desired paths for flows based on network conditions. One major limitation of host-based approaches is that every host needs to monitor the states of all paths to other hosts. In a large network such as production data centers, great amounts of control messages would occur. For many applications such as Shuffle (described in Section 5.3), each DARD host may have to monitor the entire network, which also limits its scalability. In addition, all legacy systems and applications running these protocols need to be upgraded, which incurs a lot of management cost. There are also some host-based solutions in the transport layer such as Data Center TCP (DCTCP) [13] and Multipath TCP (MPTCP) [14]. These methods are out of the scope of this work because we only focus on switching methods protocols.

- The last type is switch-only protocols which is efficient and fully compatible with current systems and applications on end hosts. It has been argued that switchonly solutions hold the best promise for dealing with large-scale and dynamic data center traffic patterns [9]. ECMP is a typical switch-only routing protocol for load balance. Many existing switch-only protocols allow a flow take multiple paths at the same time (called flow splitting) to achieve high throughput $[15,9,5]$. Flow splitting may cause a high level of TCP packet reordering, resulting in throughput drop [16].

In this paper, we propose Distributed Flow Scheduling (DiFS), a switch-only switching method that is executed independently on the control unit of each switch. DiFS aims to balance flows among different links and improves bandwidth utilization for data center networks. DiFS does not need centralized control or changes on end hosts. In addition, DiFS does not allow flow splitting and hence limits packet reordering.

Based on our observations, we categorize flow collisions in a hierarchical data center networks in two types, local and remote flow collisions. DiFS achieves load balancing by taking efforts in two directions. First, each switch uses the Path Allocation algorithm that assigns flows evenly to all outgoing links to avoid local flow collisions. Second, each switch also monitors its incoming links by running the Imbalance Detection algorithm. If a collision is detected, the switch will send an Explicit Adaption Request (EAR) message that sug- gests the sending switch of a flow to change its path. Upon receiving the EAR, the sending switch will run the Explicit Adaption algorithm to avoid remote flow collisions. Previous solutions such as Hedera [8] try to maximize the total achieved throughput across all elephant flows using global knowledge by balancing traffic load among core switches. However, we show that load balance among core switches is not enough to achieve load balance among different links. DiFS effectively solves this problem using the control messages called Explicit Adaptation Requests.

We conduct extensive simulations to compare DiFS with three representative methods from different categories: ECMP (switch-only) [6], Hedera (centralized) [8], and Dard (host-based) [3]. Experimental results show that DiFS outperforms ECMP significantly in aggregate bisection bandwidth. Compared with the centralized solution Hedera and the host-based solution Dard, DiFS achieves comparable or even higher throughput and less out-of-order packets, for both small and large data center network topologies.

The rest of this paper is organized as follows. Section 2 introduces background knowledge of flow scheduling in data center networks. Section 3 presents the detailed architecture and algorithm design of DiFS. We describe how DiFS handles network failures in Section 4. We evaluate the performance of DiFS and compare it with other solutions in Section 5. We conclude our work in Section 7.

\section{Background and Overview of DiFS}

\subsection{Data center topologies}

Today's data center networks often use multi-rooted hierarchical tree topologies (e.g., the FatTree topology [1]) to provide multiple parallel paths between any pair of hosts to enhance the network bisection bandwidth, instead of using expensive high-speed routers/switches. Our protocol DiFS is designed for a FatTree topology as long as the switch organization in every pod is the same. However for the ease of exposition and comparison with existing protocols, we will use the FatTree topology for our protocol description and simulation evaluation.

A multi-rooted hierarchical tree topology has several vertical layers. DiFS is specifically designed for 3-layer FatTree topologies. The three layers are: the edge layer, the aggregate layer, and the core layer. A pod is a management unit down from the core layer, which consists of a set of interconnected end hosts and a set of edge and aggregate switches that connect these hosts. As illustrated in Figure 1, a FatTree network is 


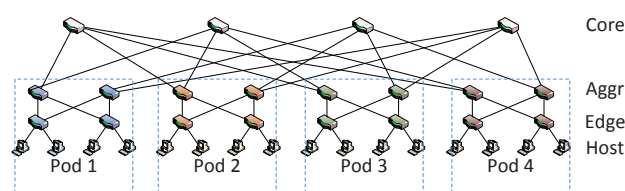

Figure 1: A fat tree topology for a datacenter network

built from a large number of $k$-port switches and end hosts. There are $k$ pods, interconnected by $(k / 2)^{2}$ core switches. Every pod consists of $(k / 2)$ edge switches and $(k / 2)$ aggregate switches. Each edge switch also connects $(k / 2)$ end hosts. In the example of Figure $1, k=4$, and thus there are four pods, each of which consists of four switches.

A path is a set of links that connect two end hosts. There are two kinds of paths in a FatTree network: interpod path and intra-pod path. An intra-pod path interconnects two hosts within the same pod while an inter-pod path is a path that connects two end host in different pods. Between any pair of end hosts in different pods, there are $(k / 2)^{2}$ equal-cost paths, each of which corresponds to a core switch. An end-to-end path can be split into two flow segments [17]. The uphill segment refers to the part of the path connecting source host to the switch in the highest layer (e.g., the core switch for an inter-pod path). The downhill segment refers to the part connecting the switch in the highest layer to the destination host. Similar to existing work, we mainly focus our discussion on inter-pod flows, because intrapod flows can be handled by a simpler version of the switching method.

In this paper we limit our discussion to FatTree. It is because that FatTree is a representative topology of hierarchical tree topologies, which have been used in the state-of-art data center designs for years [1].

\subsection{Examples of flow collision and DiFS's solutions}

DiFS aims to avoid too many flows traversing through the same switch in the network. In order to do this, each DiFS switch tries to balance the traffic across its ports and distribute the traffic among the links in the network. When too many flows are transmitted to the same link, congestion may occur in the network. Collision in the network refers to multiple flows being transmitted to the same switch, which may cause congested links. We show three types of flow collisions in Figure 2, where in each example some parts of the network are not shown for simplicity. If a switch experiences a flow collision on one of its links and can locally adjust the flow assignment to resolve the collision, such collision is called a local collision. Otherwise, the collision is called a remote collision. Figure 2 a shows an example of a local collision, where switch $A g g r_{11}$ forwards two flows to the same link. Local collisions may be caused by a bad flow assignment of static multi-pathing algorithms such as ECMP. Figure $2 b$ shows an example of Type 1 remote collision, where two flows take a same link from $\mathrm{Core}_{2}$ to $\mathrm{Pod}_{2}$. Type 1 remote collision may be caused by over-utilizing a core switch $\left(\right.$ Core $_{2}$ in this example). Hence, some existing solutions propose to balance traffic among cores [8]. However balancing core utilization may not be enough. Another example of remote collision (Type 2) is shown in Figure 2c, where core utilization is balanced but flows still collide on the link from $A_{g g r} 2$ to $E d g e_{21}$. We also observe that location collisions happen in uphill segments, and remote collisions happen in downhill segments.

Local collisions can be detected and resolved by local algorithms in a relatively easy way. DiFS uses the Path Allocation algorithm to detect flow-to-link imbalance and move one of the flows to an under-utilized link, as shown in Figure 3a. The key insight of DiFS to resolve remote collisions is to allow the switch in the downhill segment that detected flow imbalance to send an Explicit Adaption Request (EAR) message to the uphill segment. The concept of EAR is introduced in [5]. For the example of Figure 2b, $\operatorname{Aggr}_{21}$ can detect flow imbalance among the incoming links. It then sends an EAR to $\mathrm{Aggr}_{31}$ in $\mathrm{Pod}_{3}$ (randomly chosen between two sending pods), suggesting the flow to take the path through Core $_{1}$. Aggr 31 runs the Explicit Adaption algorithm and changes the flow path. That flow will eventually take another incoming link of Aggr $_{21}$ as shown in Figure 3b. To resolve the collision in Figure $2 \mathrm{c}, E d g e_{21}$ that detects flow imbalance sends back an EAR and suggest $E d g e_{12}$ to forward the flow to $A g g r_{11}$. That flow will eventually go from $A g g r_{21}$ to $E d g e_{21}$, as shown in Figure 3c.

The incoming links of the aggregate (edge) switch in the downhill segment have a one-to-one correspondence to the outgoing links of the aggregate (edge) switch in the uphill segment in a multi-rooted tree. This is consistent with the observations in [18]. Therefore, when an aggregate (edge) switch in the downhill segment detects imbalance and finds an under-utilized link, it can suggest the aggregate (edge) switch in the uphill segment to change the path to the "mirror" of the under-utilized link. In the example of Type 1 remote collision, Aggr $_{21}$ controls the flow to income from Core $_{1}$ by suggesting $\mathrm{Aggr}_{31}$ to forward the flow to Core $_{1}$. In the example of Type 2 remote collision, $E d g e_{21}$ controls the flow to income from $A g g r_{21}$ by suggesting $E d g e_{12}$ to forward the flow to $A g g r_{11}$. 


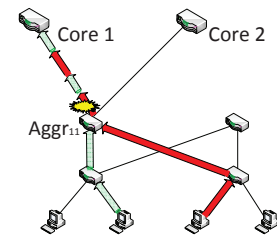

(a) Local collision

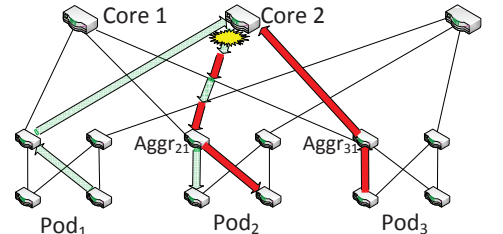

(b) Optimal algorithms for doubly weighted approximation of univariate functions

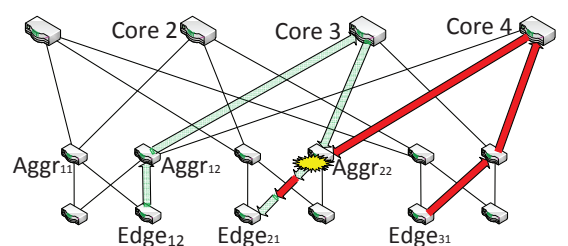

(c) Remote collision Type 2: load balance among core switches is not enough

Figure 2: Three types of collisions. For simplicity we use two flows to indicate a collision. In practise, a collision may be caused by many flows.

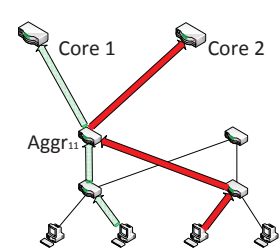

(a) Path Allocation solves local collision.

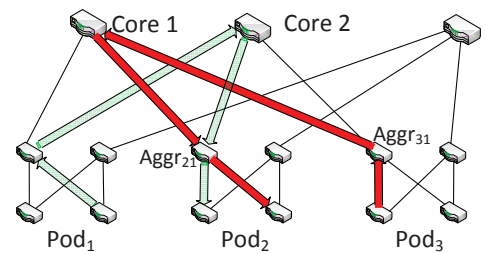

(b) Solve remote collision Type 1: Aggr $_{21}$ sends back an EAR and suggest Aggr $_{31}$ to forward the flow to Core 1 .

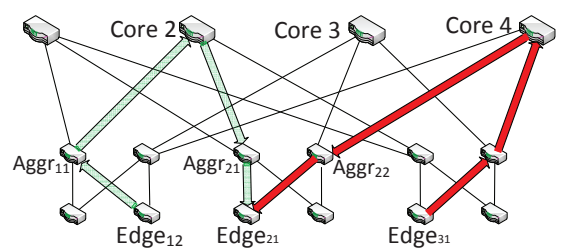

(c) Solve remote collision Type 2: $E d g e_{21}$ sends back an EAR and suggest $E d g e_{12}$ to forward the flow to Aggr11.

Figure 3: Resolving collisions by Path Allocation and Explicit Adaption

\subsection{Classification of flows}

In this paper, a flow is defined as a sequence of packets sent from a source host to a destination host using TCP. In our flow scheduling protocol, a flow can have only one path at any time. Allowing a flow to use multiple paths simultaneously may cause packet reordering and hence reduce the throughput. However, a flow is allowed to take multiple paths at different times in its life cycle.

Elephant and mice flows: DiFS considers the traffic in the network as elephant flows and mice flows. In this work, elephant flows are defined as large, long-lived flows. The traffic volume of these flows is significantly larger than that of mice flows. Typical examples of elephant flows include virtual machine migration traffic, traffic of data mining applications and log file backup traffic. The other flows are called mice flows. Similar to many other works $[8,3]$, our protocol focuses on elephant flows and intends to spread them as evenly as possible among all links. All mice flows will be processed by ECMP, because recent work has shown that ECMP forwarding can perform load-balancing efficiently and effectively for mice flows [4]. Note that at a particular time, the sending rate of an elephant flow is not necessarily larger than that of a mice flow. Whether a flow is an elephant or not is determined by the overall traffic volume.

Classification of elephant flows: Let $f_{a b}$ be a flow whose source is $a$ and destination is $b$. A flow $f_{a b}$ may be classified into four types for a particular switch $s$ that runs DiFS:

- $f_{a b}$ is a single-in-single-out (SISO) flow for switch $s$ if and only if there are only one possible incoming link of $s$ from $a$ and one possible outgoing link of $s$ to $b$. In Figure 4, the core switch Core 2 considers the flow from Host 21 to Host $_{31}$ (illustrated as a red line) is a SISO flow.

- $f_{a b}$ is a single-in-multi-out (SIMO) flow for switch $s$ if and only if there are one incoming link of $s$ from $a$ and multiple outgoing links of $s$ to $b$. In Figure 4, dge $_{21}$ considers the illustrated flow is a SIMO flow.

- $f_{a b}$ is a multi-in-single-out (MISO) flow for switch $s$ if and only if there are multiple incoming links of $s$ from $a$ and one outgoing link of $s$ to $b$. A close look at FatTree networks reveals that all interpod flows are SIMO for the edge and aggregate switches on the uphill segments, and are MISO for the edge and aggregate switches on the downhill segments. All inter-pod flows for core switches are SISO. In Figure 4, ggrr $_{31}$ considers the illustrated flow is a MISO flow. is a MISO flow because the switch can only forward this flow in one link but this flow can choose different cores so that it can 


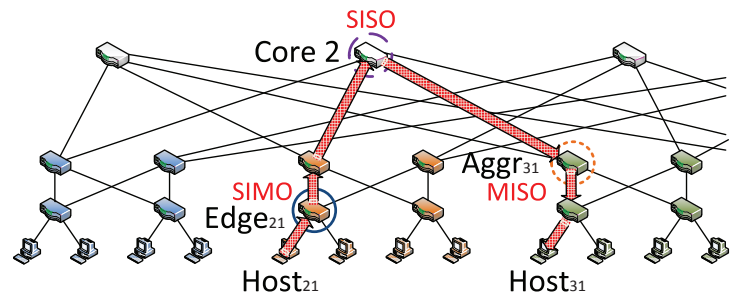

Figure 4: Examples of SISO, SIMO and MISO flows

come into the aggregate switch from more than one port.

- Multi-in-multi-out (MIMO) flows may be defined similarly. However, there is no MIMO flow for any switch in a FatTree network. They may appear in general topologies.

Note one flow may be treated as different types by different switches.

Look at the flow from Host Ho $_{21}$ to Host $_{31}$ in Figure 4. For edge switch $E d g e_{21}$, this is SIMO flow since it has 2 output links to forward it. For Core2, this is a SISO flow as it can only be forwarded to Pod3. For Aggregate switch $\operatorname{Aggr}_{31}$, this flow is a MISO flow as the flow can be forwarded by another connected core switch.

\section{DiFS Design}

\subsection{Components and Deployment}

A typical switch architecture usually consists of two components: data plane and control plane. The data plane includes multiple network ports, as well as a flow/forwarding table and an output queue for each port. The control plane can perform general-purpose processing like collecting measurement results and install/modify the rules in the flow/forwarding tables of the data plane. As a result, DiFS should be installed in the control plane of each switch.

Requirement of the switches. In order to execute the DiFS operations, the switches should be able to: (1) Identify elephant flows from all the flows that traverse through the switch. (2) In its local memory, maintain three variables for each elephant flow $f: L_{i}, L_{o}$ and $t$; maintain two integer vectors that describes the switch status, $V_{i}$ and $V_{o}$. DiFS uses these two vectors to measure the load of a switch, not other information such as CPU utilization ratio. (3) Execute a control loop. In each loop, perform the instructions defined in Algorithm 1 (see Section 3.4). (3) Execute the given algorithm described in Algorithm 2 and Algorithm 3 upon receiving control messages EAR and PAR. The DiFS switches should be able to execute arithmetic and logic operations, and send out control messages based on the execution results. Modern enterprise or data centers usually use high-end switches. These switches are usually equipped with powerful CPUs and fast local memory. Some typical representatives include the Cisco Catalyst series [19] and Hewlett-Packard Enterprise FlexNetwork series [20]. Hence, we believe DiFS can be implemented on these switches.

Implementation on generic platforms. We notice the trend of using software-based switches with commodity hardware in data centers [21, 22]. These switches are commodity computers (e.g., x86_64 systems) equipped with high-end Network Interface Cards (NICs). The DiFS algorithm on a switch can be implemented as a program running on a computer. We recommend using the Intel Data Plane Development Kit (DPDK) [23]. DPDK is a set of software libraries that allow a Linux system to process packets effectively. DiFS can be implemented as a DPDK application. This application program reads packets from the Tx queues of the NICs, executes DiFS algorithms, and then forward the packet to the corresponding $\mathbf{R x}$ queues.

Operating with SDN. DiFS is also compatible to software defined networking such as OpenFlow [7]. For each switch in the network, DiFS distributes the flows among the links in order to achieve global flow balance and high throughput. In SDN, the controller decides the behavior of individual flows. The control logic of DiFS can be implemented in the controllers. The controller executes DiFS algorithms and decides the paths of all flows in the network. The controller communicates with the switches to install the flow tables so that the switches may forward the traffic as instructed.

Compared with centralized algorithms that require a single controller responsible for the entire network, distributed and localized decision-making of DiFS offers tremendous scalability to SDN control. For example, OpenFlow switches in the same pod can be connected to one controller, which is physically close to these switches and able to handle the scheduling tasks. The communication overhead of the control is much smaller when local controllers are deployed than when the network uses one central controller. 


\subsection{Optimization goals}

As a high-level description, DiFS intends to balance the number of elephant flows among all links in the network to utilize the bisection bandwidth and take the advantage of path diversity. We use the number of flows as the optimization metric instead of flow bandwidth consumption based on the following reasons:

1. A flow's maximum bandwidth consumption ${ }^{4}$ can hardly be estimated. As shown in [8], a flow's current sending rate tells very little about its maximum bandwidth consumption. Hedera [8] uses global knowledge to perform flow bandwidth demand estimation. However, such method is not possible to be applied in distributed algorithms such as DiFS.

2. Using flow count only requires a switch to maintain a counter for each outgoing link. However, measurement of flow bandwidth consumption requires complicated traffic monitoring tools installed on each switch. Our method simplifies switch structure.

3. Using flow count as the metric, DiFS can achieve similar or even better performance compared with Hedera [8] and a variant of DiFS implementation that uses estimated bandwidth consumption as the metric. The results will be shown in Section 5.7.

If the elephant flows are sufficiently large, and each host makes its best effort to transmit packets, the number of elephant flows is proportional to the bandwidth utilized by these flows.

Two optimization goals for load-balancing scenarios are desired:

Balanced Output (BO): For an edge switch $s_{e}$, let $o\left(s_{a}\right)$ be the number of SIMO flows on an outgoing link connecting the aggregate switch $s_{a}$. BO of edge switch $s_{e}$ is achieved if and only if $o\left(s_{a 1}\right)-o\left(s_{a 2}\right) \leq \delta$, for any two aggregate switches $s_{a 1}$ and $s_{a 2}$, where $\delta$ is a constant. Similarly we may define BO of an aggregate switch to cores. BO can be achieved by the Path allocation algorithm of DiFS with the smallest possible value of $\delta$ being 1 .

Balanced Input (BI): For an aggregate switch $s_{a}$, let $i(c)$ be the number of MISO flows on an incoming link connecting the core $c$. BI of edge switch $s$ is achieved if and only if $i\left(c_{1}\right)-i\left(c_{2}\right) \leq \delta$, for any two cores $c_{1}$ and $c_{2}$, where $\delta$ is a constant. Similarly, we may define BI of an edge switch from aggregate switches. BI can

\footnotetext{
${ }^{4}$ A flow's maximum bandwidth consumption, also called as flow demand, is the rate the flow would grow to in a fully non-blocking network.
}

be achieved by Explicit Adaptation of DiFS with the smallest possible value of $\delta$ being 1 .

$\mathrm{BO}$ and $\mathrm{BI}$ do not interfere with each other. Hence, a switch can achieve them at the same time. Although $\mathrm{BO}$ and $\mathrm{BI}$ of a switch are two kinds of optimization in a local view, we have proved that they provide global performance bounds of load balancing, as presented in Section 3.7. In Section 5 we further demonstrate that they can achieve high aggregate throughput via simulations.

\subsection{Protocol Structure}

DiFS uses a threshold to eliminate mice flows. Such threshold-based module can be installed on edge switches. It maintains the number of transmitted bytes of each flow. This monitoring task can be cost-efficient in switch resources using recently proposed techniques such as OpenSketch [24]. If the byte number of a flow is larger than a threshold value, the edge switch will label this flow as an elephant flow and mark the packet header to notify other switches on its path.

Each switch has a flow list which maintains three variables for every flow $f$ : the incoming link identifier, denoted as $L_{i}$, the outgoing link identifier, denoted as $L_{o}$, and the last time this flow appeared, denoted as $t$. A switch also maintains two Port State Vectors (PSVs), $V_{i}$ and $V_{o}$. The $i$ th element in vector $V_{i}$ is the number of flows coming from the $i$ th incoming link. Likewise the $i$ th element in vector $V_{o}$ is the number of flows forwarded to the $i$ th outgoing link.

There are three flow control modules in aggregate and edge switches: control loop unit, explicit adaptation unit, and path allocation unit. Control loops are run periodically by switches. The main objectives of the control loop unit are to detect imbalance of MISO flows among incoming links and send an Explicit Adaptation Request (EAR) if necessary. An EAR is a notification message sent along the reverse flow path to recommend switches in the flow's sending pod to choose a different path. An EAR also includes a path recommendation. When a switch receives an EAR, it runs the explicit adaptation unit and changes the output link of the designated flow in the EAR to that on the recommended path, if possible. Path Allocation Request (PAR) is another message to request flow scheduling. PAR includes a flow identifier and requires switches to allocate an available link for this flow. Switches treat a packet with a new flow identifier as a PAR. The sender needs to explicitly send a PAR only if path reservation is allowed to achieve a certain level of performance guarantee for upper-layer applications [25]. For a SIMO flow, the path allocation unit will assign an outgoing port for this flow 


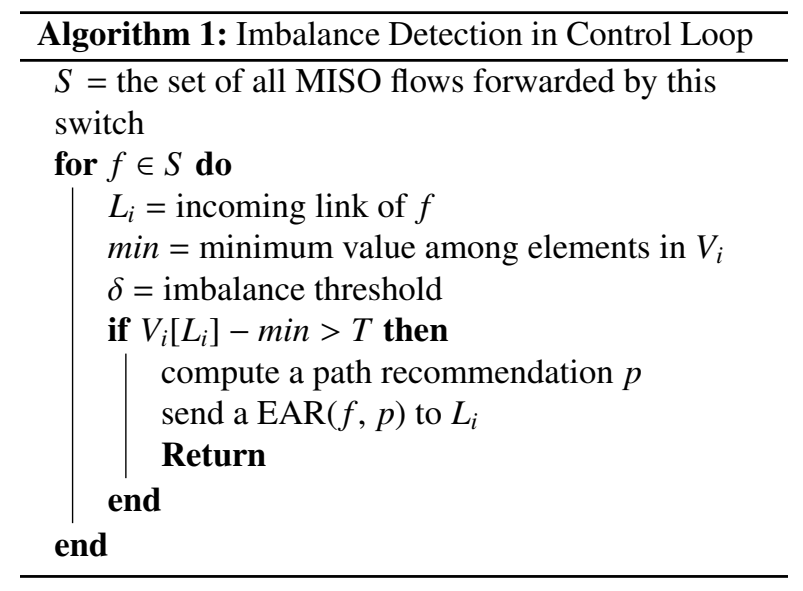

based on link utilization. Detailed algorithms for these modules will be presented in the following subsections.

The time period between two control loops has limited impact on the convergence time of the whole protocol execution. We will show that DiFS converges quickly under a wide range of control loop period time in Section 5.5.

\subsection{Control loop}

Each DiFS switch continuously runs a control loop. At each iteration, the switch executes the following:

1. Remove disappeared flows. A flow may disappear from a switch due to several reasons. For example, the flow may have finished transmission or taken another path. In each iteration, the switch will delete a flow if the difference between current time and its last-appeared time $t$ is larger than a threshold, which may be set to a multiple of the average round-trip time of flows.

2. Re-balance SIMO flows among all outgoing links. Removing disappeared flows may cause the change of flow numbers on links. Thus flow rebalancing is necessary.

3. Send an EAR if necessary. If the switch finds a MISO flow comes in a over-utilized link, the switch will recommend other switches to change the flow path by sending an EAR. In order to avoid TCP performance degrade caused by too many EARs, DiFS forces every switch to send at most one EAR at each iteration.

We detail the steps 2) and 3) as the follows.

Re-balance SIMO flows. The purpose of rebalancing SIMO flows is to achieve BO, i.e., let the flow count difference of any two outgoing links be smaller than the pre-defined threshold $\delta$. The solution seems to be trivial: a switch can simply move flows on overloaded links to under-loaded ones. However, this simple method could cause oscillations of network status. Consider a switch $s$ moves a random flow $f$ from link $l_{1}$ to $l_{2}$ for load balance. Later by receiving an EAR from another switch, $s$ will be suggested to move $f$ from $l_{2}$ to $l_{1}$ to avoid remote collisions. During the next control loop, $s$ will again move $f$ to $l_{1}$ to $l_{2}$ and so on. Such oscillation will never stop. One obvious downside of oscillations is that they will incur packet reordering and hurt TCP performance. To resolve this problem, we maintain a priority value for each flow in the flow list. When the link assignment of a flow is changed based on the suggestion from an EAR, the priority of the flow is increased by one. When a switch re-balances SIMO flows, it should first move flows that have smaller priority values. This strategy intends to let flows whose assignments are changed by EARs be more stable and reduce the probability of oscillations, by which the system convergence will be faster.

Imbalance detection and path recommendation for EAR. For fairness concern, at each iteration, the switch will scan each MISO flows in a random order. The imbalance detection is also in a threshold basis, which is presented in Algorithm 1.

Due to lack of global view of flow distribution, the EAR receiver should be told how to change the flow's path. Therefore, the EAR sender should include a path recommendation, which does not necessarily need to be a complete path. In a FatTree, both aggregate and edge switches can detect load imbalance and recommend an alternative path only based on local link status.

For the flow collision example of Figure 2b, Aggr $_{21}$ will notice the load imbalance among incoming links and send an EAR to Aggr $_{31}$ (randomly selected between senders of the two collided flows). The path recommendation in this EAR is just Core $_{1}$. Aggr 31 will receive the EAR and change the flow to the output link connected with Core $_{1}$, and this flow will eventually come from another incoming link of $A g g r_{21}$ that was under-utilized, as shown in Figure 3b.

For the flow collision example of Figure 2c, Edge 21 can detect it by comparing two incoming links and then send an EAR to $E d g e_{12}$ in the uphill segment. The path recommendation here is just $A g g r_{11}$. When $E d g e_{12}$ let the flow take $A g g r_{11}$, the flow will eventually take another incoming link to $E d g e_{21}$ and hence resolves the collision as shown in Figure 3c.

As a matter of fact, in a FatTree network, a path recommendation can be specified by either a recommended core or a recommended aggregate switch in the uphill segment. For other topologies, more detailed path spec- 


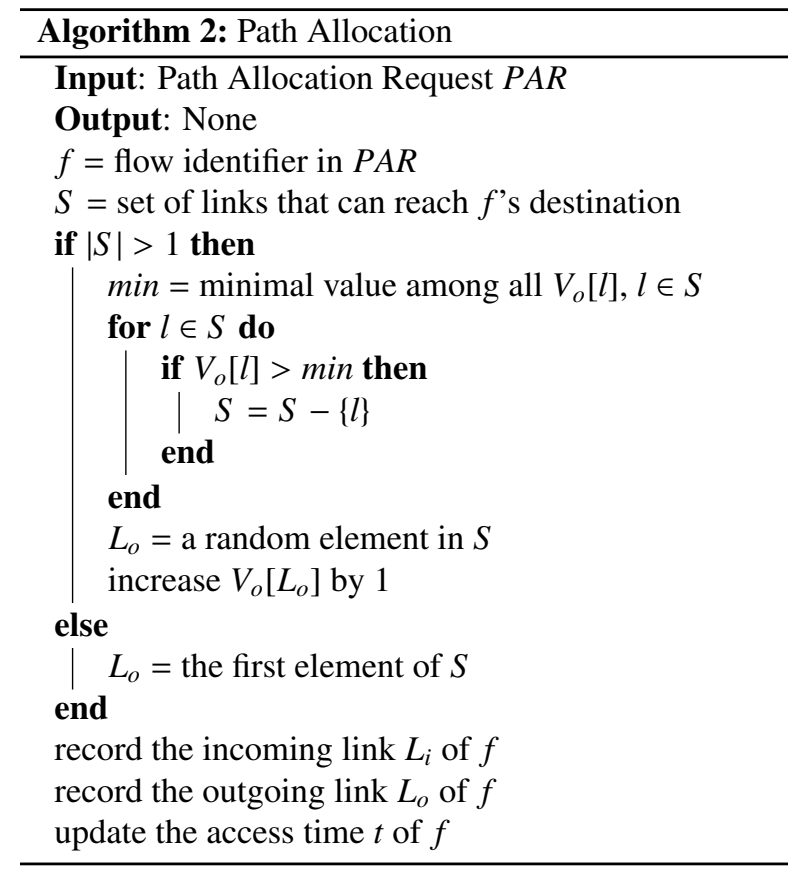

ification might be needed.

For an intra-pod flow, the path consists of two edge switches and one aggregate switch. If the aggregate switch detects load imbalance, it can also send an EAR to the edge switch in the previous hop and suggest the edge switch to send the flow to another aggregate switch. In fact, this is the one difference in our protocol when it treats intra-pod and inter-pod flows.

\subsection{Operations upon receiving a PAR}

As presented in Algorithm 2, To keep all links output balanced, we use a distributed greedy algorithm to select an outgoing link for each flow requested by a PAR. When a switch received a PAR, it first checks how many outgoing links can lead to the destination. If there is only one link, then the switch will simply use this link. If there are multiple links to which this flow can be forwarded, the switch will select a local optimal link for this flow. The algorithm first find the set of links with the minimum number of outgoing flows. If there are more than one links in this set, the algorithm will randomly select a link from the set.

\subsection{Operations upon receiving an EAR}

An EAR includes a flow identifier and a path recommendation. As mentioned, for a FatTree network a path recommendation can be specified by either a recommended core or a recommended uphill aggregate

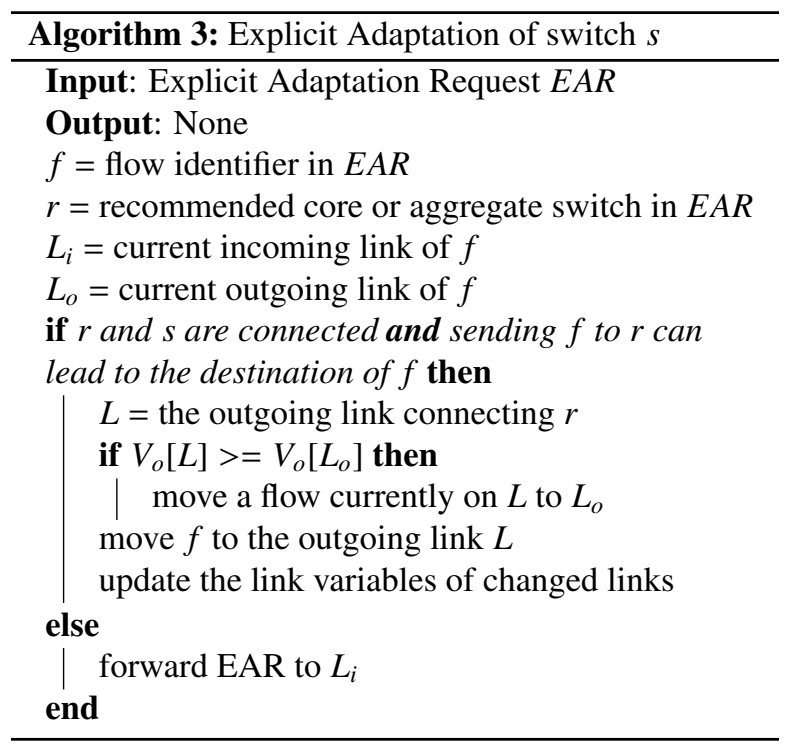

switch. When a switch received an EAR, it first checks if it can move the requested flow $f$ to the recommended core or aggregate switch. If not, it will forward this EAR further towards the reverse path of $f$. If moving $f$ will cause imbalance among outgoing links, the switch swaps $f$ with another flow on the recommended link. The complete algorithm is described in Algorithm 3.

EARs may also cause network status oscillations. Consider the following scenario in Fig 5, where only part of the network is shown. flow 1 and flow 2 collide on the same link from $S W_{4}$ to $S W_{1}$ but the link from $S W_{5}$ to $S W_{1}$ is free. $S W_{1}$ may send an EAR to $S W_{2}$ and suggest $S W_{2}$ to send flow 1 to $S W_{5}$, in the purpose of resolving the remote collision at $S W_{1}$. After receiving the EAR, $S W_{2}$ swaps the outgoing links of flow and flow $_{3}$. However at the same time $S W_{3}$ may send an EAR to $S W_{2}$ and suggest $S W_{2}$ to send flow 4 to $S W_{4}$. $S W_{2}$ should then swap the outgoing links of flow 2 and flow $_{4}$. As a result the collisions still exist. By keeping executing the protocol, oscillations happen and the network status cannot converge. To deal with the problem, we allow random spans in control loops. There is some non-negligible time difference between the control loops of $S W_{1}$ and $S W_{3}$. In this way, $S W_{3}$ may notice that its collision has already been solved after $S W_{2}$ swaps the outgoing links of flow 1 and flow flo $_{3}$ and will not send another EAR.

\subsection{Bounds on global flow balance}

The local optimization on switches can lead to global performance bounds as introduced in this section. 


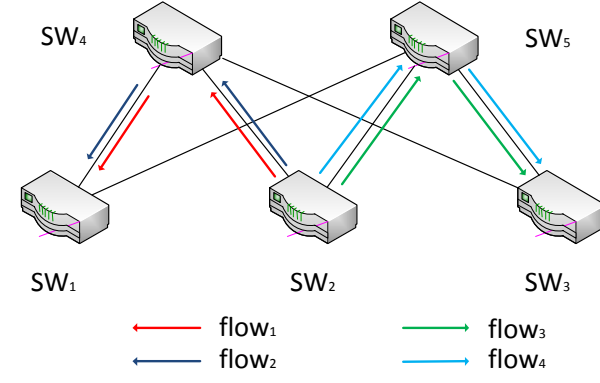

Figure 5: Oscillation problem caused by EARs

We provide a bound on flow balance among aggregate switches in a same pod by the following theorem:

Theorem 3.1. In a k-pod FatTree, suppose every edge switch achieves $B O$ with $\delta$. Let $n\left(s_{a}\right)$ be the number of flows that are sending to aggregate switch $s_{a}$. Then we have $M A X_{a}-M I N_{a} \leq \delta \cdot k / 2$, where $M A X_{a}$ is the maximum $n\left(s_{a}\right)$ value among all aggregate switches in the pod, $M I N_{c}$ is the minimum $n\left(s_{a}\right)$ value among all aggregate switches in the pod.

Proof. Let $x$ and $y$ be arbitrary two aggregate switches. Let $n_{a e}$ be the number of flows from edge switch $e$ to aggregate switch $a$.

$$
\begin{aligned}
& n(x)=\sum n_{x e} \\
& n(y)=\sum n_{y e}
\end{aligned}
$$

Since $\left|n_{x e}-n_{y e}\right| \leq \delta$ for every edge switches $e$ and there are $k / 2$ edge switches in a pod,

$$
|n(x)-n(y)| \leq \sum\left|n_{x e}-n_{y e}\right| \leq \delta \cdot k / 2
$$

Hence $M A X_{a}-M I N_{a} \leq \delta \cdot k / 2$.

We further prove a bound on flow balance among core switches by the following theorem:

Theorem 3.2. In a k-pod FatTree, suppose every edge and aggregate switch achieves $B O$ with $\delta=1$. Let $n(c)$ be the number of flows that are sending to core $c$. Then we have $M A X_{\text {all }}-M I N_{\text {all }} \leq 3 k$, where $M A X_{\text {all }}$ is the maximum $n(c)$ value among all cores and $M I N_{\text {all }}$ is the minimum $n(c)$ value among all cores.

Proof. The $(k / 2)^{2}$ cores can be divided into $k / 2$ groups $g_{1}, g_{2}, \ldots, g_{k / 2}$, each of which contains $k / 2$ cores that receive flows from a same group of aggregate switches.

Suppose $x$ and $y$ are two cores. If they belong to a same group, we can prove $n_{x}-n_{y} \leq k / 2$ using a way similar to the proof of Theorem 3.1. Consider that they belong to different groups. For a pod $p, x$ and $y$ connect to two different switches in $p$, because they are in different core groups. Let $s_{a 1}$ and $s_{a 2}$ denote the switches connecting to $x$ and $y$ respectively. We have $n\left(s_{a 1}\right)-n\left(s_{a 2}\right) \leq k / 2$ according to Theorem 3.1. Hence

$$
\frac{n\left(s_{a 1}\right)}{k / 2}-\frac{n\left(s_{a 2}\right)}{k / 2} \leq 1
$$

Hence, the average numbers of flows from $s_{a 1}$ and $s_{a 2}$ to each core are $\frac{n\left(s_{a 1}\right)}{k / 2}$ and $\frac{n\left(s_{a 2}\right)}{k / 2}$ respectively. Let $n_{p c}$ denote the number of flows from pod $p$ to core $c$. We have $n_{p x}-\frac{n\left(s_{a 1}\right)}{k / 2} \leq 1$ ( $\mathrm{BO}$ of $\left.s_{a 1}\right)$, and $\frac{n\left(s_{a 2}\right)}{k / 2}-n_{p y} \leq 1$ (BO of $s_{a 2}$ ). Hence

$$
\begin{aligned}
& n_{p x}-n_{p y} \leq 1+\frac{n\left(s_{a 1}\right)}{k / 2}-\frac{n\left(s_{a 2}\right)}{k / 2}+1 \leq 3 \\
& n_{x}-n_{y}=\sum_{p} n_{p x}-\sum_{p} n_{p y}=\sum_{p}\left(n_{p x}-n_{p y}\right) \leq 3 k
\end{aligned}
$$

Similarly we have a bound of flow balance in the receiving side.

Theorem 3.3. In a k-pod FatTree, suppose all aggregate switches in a same pod achieve BI with $\delta=1$. Let $n\left(s_{e}\right)$ be the number of flows that are sending to edge switch $s_{e}$. Then we have $M A X_{e}-M I N_{e} \leq k / 2$, where $M A X_{e}$ is the maximum $n\left(s_{e}\right)$ value among all edge switches in the pod and $M I N_{e}$ is the minimum $n\left(s_{e}\right)$ value among all edge switches in the pod.

The proof is similar to that of Theorem 3.1.

Note that the values we provide in the theorems are only bounds of the difference between the maximum and minimum flow numbers. In practice, however, the actual differences are much lower than these bounds.

\subsection{Impact on mice flows}

DiFS aims to balance the traffic of elephant flows. DiFS moves flows from highly occupied links to links with abundant free bandwidth. As presented above, DiFS balances elephant flows among aggregate switches. Consider a mice flow that goes through links $L_{1}, L_{2}, \cdots, L_{n}$ and switches $S_{1}, S_{2}, \cdots, S_{n-1}$. The maximum transmission rate of this flow depends on the available bandwidth and computation resource of these links and switches. Particularly, it depends on the minimal available bandwidth of all $n$ links. Suppose the available bandwidth on link $L_{i}$ is $r_{i}>0$. Clearly, when $\sigma r_{i}$ is constant, a more uniformly distributed $r_{1}, r_{2}, \cdots, r_{n}$ would result in a larger $\min r_{i}$. This is to say when the traffic is uniformly distributed, the available bandwidth of a mice flow is likely to be larger. 
Mice flows in the network can be categorized into two groups. (1) low bandwidth and long-lasting. (2) short burst flows. Flows in (1) do not require large resources. Meanwhile, DiFS gives favor to flows in (2) by balancing the traffic of the elephant flows.

\subsection{Flow termination}

Most switches support flow expiration. i.e., the OpenFlow standard specifies that for each flow entry on the switch, there is an idle time-out value that indicates when this entry shall be removed due to a lack of inactivity. When a flow entry is expired and removed, we consider the corresponding flow terminates.

\subsection{Switch overhead of DiFS}

In DiFS, each switch maintains a list of elephant flows, and two vectors $V_{i}$ and $V_{o}$. Executing the DiFS algorithms consumes computation resources. We analyze the time overhead of DiFS algorithms as follows.

The switch maintains integer vectors $V_{i}$ and $V_{o}$. It often computes the minimal value of $V_{i}$ and $V_{o} . V_{i}$ and $V_{o}$ can be implemented in integer arrays with $O(n)$ for each query and $O(1)$ for each update. However, we recommend using a heap or binary index tree with $O(\log n)$ for all operations. Note that the lengths of $V_{i}$ and $V_{o}$ are both equal to the number of ports of the switch, which is a small constant. This is to say $V_{i}$ and $V_{o}$ can be maintained effectively in constant time.

The algorithm within one iteration in the control loop includes detecting the imbalance, re-balancing, and sending EAR if necessary. The time complexity is at most $O(n)$ where $n$ is the number of elephant flows on the switch. This would take several milliseconds when there are thousands of elephant flows. Experiments show that DiFS is able to converge even with control loops for every $100 \mathrm{~ms}$. Hence, the time overhead of the instructions executed in one loop is sufficiently small.

On receiving an EAR, the switch executes the particular algorithm with time complexity $O(1)$. On receiving a PAR, the time complexity is at most $O(p)$ where $p$ is the number of ports on the switch. These two algorithms only consist of simple arithmetic operations and can be executed within several hundreds of CPU cycles. This is to say the procedure is able to finish within $1 \mu$ s on an $1 \mathrm{GHz} \mathrm{CPU}$, which is acceptable for most commodity switches.

\section{Failures Recovery}

Switches must take network failures into consideration in performing flow scheduling. A network failure may be a switch failure, a link failure, or a host failure. Failures may also be classified into reachability failures and partial failures. Reachability failures refer to those failures that can cause one or more end hosts unreachable. For example, a crash of an edge switch can make $(k / 2)$ hosts unreachable. We propose mechanisms for DiFS to maintain network performance under these failures. Only flows towards the unreachable hosts are affected.

Partial failures, i.e., individual link or port failures on edge and aggregate switches, can cause performance degradation due to loss of equal-cost paths. We classify partial failures into two main types: Independent Link Failures (ILFs) and Compositional Failures (CFs). ILFs are link or port failures that are independent of each other, i.e., no two link or port failures collide on the same switch. The most frequent independent link failures are single link failures. CFs are referred to node failures and multiple link failures that can occur on the same switch node. Although CFs may cause more severe results, they happen very rarely compared to ILFs [26].

Since ILFs are very common in Data Center Networks, we first introduce how DiFS deals with them. When a link is down, the switch that was connected by this link can realize it and find a longer detour that can bypass the failed link. For example, if the link connecting a core switch and an aggregate switch is failed, this failed link can be replaced by a two hop detour that still connects the core switch and aggregate switches. Such detours can be computed at a low cost by switches. Given the fact that switches know the fat tree topology, which is a reasonable assumption as the network topology does not change in a long term. Unlike Baatdaat [27] using a measurement based metric computing the lowest cost detour, DiFS will randomly select a feasible detour for each packet. Since it is rare that one packet may have two or more detours under independent link failures, detouring a packet can cause a very low cost.

Although it is very easy to compute a detour under ILFs, things are very different for CFs. Node failures and related link failures can make it harder to compute a feasible detour or even impossible to find a detour with limited hop. To avoid the complexity caused by compositional failures, we decide not to detour packets when compositional failures are detected. Instead, If an output port for some elephant flow is no longer available or cannot reach the destination due to compositional fail- 
ures, DiFS will randomly select an output port for each packet among all the feasible outgoing links. The DiFS switch that is nearest to the source of the affected flow will choose the output port for the packets. However, packets may arrive at the destination but not in the original order. This is resolved by higher level protocols such as TCP. At the same time, this flow will still be maintained in flow table but all future EARs will be ignored. After the failure is fixed, the old flow table can be quickly reused at a low cost. The switch may store the old flow table entries in its local memory and restore them when the failure is fixed. This method uses different paths for packets of the same flow and could hurt TCP performance due to packet re-ordering. However, we argue that the TCP performance has already been affected by network failures and hence packet re-ordering may be allowed under this circumstance.

Loss or delay of EARs on a congested link may make DiFS degrade into a local link balanced algorithm like ECMP. the EAR messages are sent in UDP to avoid additional costs such as the communication overhead of TCP handshaking. However, a switch will keep sending control messages at each control loop if previous flow collisions have not been resolved. In the simulations, we also take the loss and delay of control messages into consideration. Experiments show that DiFS still converges in a short time under congestion. In fact, even an EAR is missing, the network connectivity is not affected, and hence the related flow is not affected by such missing. Therefore, the loss or delay of control messages has limited impact to network convergence.

\section{Simulation Results}

In this section, we evaluate the performance of DiFS by comparing it with three representative routing solutions from different categories: ECMP (switch-only) [6], Hedera (centralized) [8], and Dard (host-based) [3]. Note that both Hedera and Dard use global network information which is not available to switch-only methods.

\subsection{Methodology}

Most existing studies use custom-built simulators to evaluate data center networks at large scale [8] [14] [28] [9]. Simulation is able to show the scalability of the protocols for large networks with dynamic traffic patterns while testbed experiments can only have up to tens of hosts for academic purposes. We find many of them use a certain level of abstraction for TCP, which may result in inaccurate throughput results. ${ }^{5}$ To perform simulations with accurate results, we developed a packet-level stand-alone simulator ${ }^{6}$ in which DiFS, as well as other protocols, are implemented in detail. ${ }^{7}$ Our simulator models individual packets, hence we believe it can better demonstrate real network performance. TCP New Reno is implemented in detail as the transportation layer protocol. Our simulator models each link as a queue whose size is the delay-bandwidth product. A link's bandwidth is $1 \mathrm{Gbps}$ and its average delay is $0.01 \mathrm{~ms}$. Our switch abstraction maintains finite shared buffers and forwarding tables. In our simulations, we simulate multi-rooted tree topologies in different sizes. We use 16-host networks as small topologies and 1024-host networks for bulk analysis.

DiFS is compared with ECMP, Hedera, and Dard. For ECMP, we implemented a simple hash function which uses the flow identifier of each TCP packet as the key. We implemented the Simulated Annealing scheduler of Hedera, which achieves the best performance among all schedulers proposed in Hedera [8]. We set the control loop period of Hedera to 0.01 seconds and Simulated Annealing iteration to 1000 , both of which are the same as their implementation. We also set the period of distributed control loop to 0.01 second for DiFS. As mentioned in Section 3.2, we focus on balancing the number of elephant flows among links. We use $100 \mathrm{~KB}$ as the elephant threshold, same to the value used by other work [3].

Performance criteria. We evaluate the following performance criteria.

Aggregate throughput is the measured throughput of various traffic patterns using proposed methods on the corresponding data center topology. It reflects how a switching method utilize the topology bandwidth.

Flow completion time characterizes the time to deliver a flow, which may affect the processing efficiency of a data center. Besides the comparison of flow completion time among different protocols, we also care about the fairness of flow completion time of different flows routed by the same protocol.

\footnotetext{
${ }^{5}$ For example, the simulator developed in [8] only simulates each flow without performing per-packet computation and uses predicted sending rate instead of implementing TCP. The simulator that implements MPTCP [14] has been used for performance evaluation by many other projects [28] [9]. However, it does not implement TCP ACKs and assumes ACKs can all be successfully delivered.

${ }^{6}$ The simulator is available via https://github.com/ sdyy 1990/NSim/raw/master/NSim.7z

${ }^{7}$ We have also implemented DiFS on NS2, but experienced very slow speed when using NS2 for data center networks. We guess the existing studies do not use NS2 due to the same reason.
} 


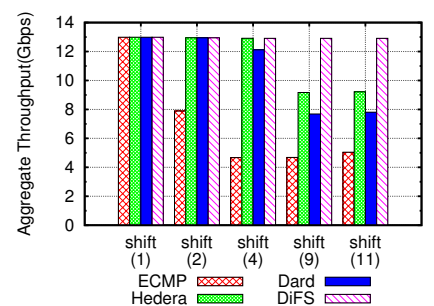

(a) Shift traffic pattern

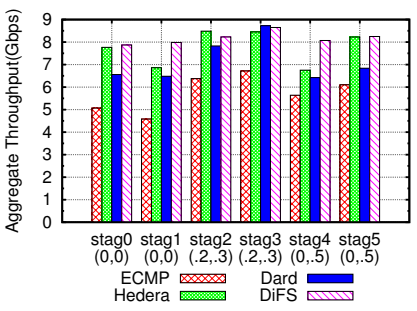

(b) Staggered traffic pattern

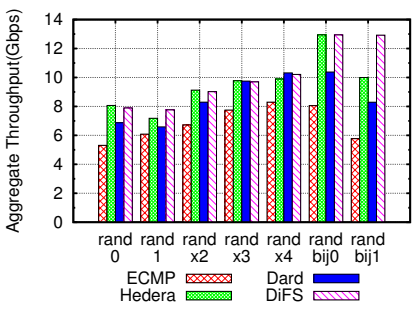

(c) Random traffic pattern

Figure 6: Aggregate throughput comparison on small topologies

Packet out-of-order ratio. Although all protocols in our simulations do not split flows, dynamic routing will still cause some out-of-order packets. The out-of-order ratio is measured to see whether a protocol will hurt TCP performance.

Convergence time is important to measure the stability of a dynamic routing protocol or switching method.

Control overhead. We measure the control message overhead in bytes.

Traffic patterns. Similar to [8] and [3], we created a group of traffic patterns as our benchmark communication suite. Each flow is at least 16MB. These patterns are considered typical for cluster computing applications and can be either static or dynamic. For static traffic patterns, all flows are permanent. Dynamic traffic patterns refer to those in which flows start at different times. In this paper, we evaluate the performance of DiFS against dynamic patterns similar to data shuffle in cluster computing applications such as MapReduce [29]. The static patterns used by our simulations are described as follows:

1. Shift(i): A host with index $x$ sends data to a host with index $(x+i) \bmod ($ num_hosts $)$, where num_hosts is the number of all hosts in the network. This traffic pattern stresses out the links between the core and the aggregation layers with a large $i$.

2. Staggered $\left(P_{e}, P_{p}\right)$ : A host sends data to another host in the same edge layer with probability $P_{e}$, and to host in the same pod (but in the different edge layer) with probability $P_{p}$, and to hosts in different pods with probability $1-P_{e}-P_{p}$.

3. Random: A host sends one elephant flow to some other end host in the same network with a uniform probability. This is a special case of $\operatorname{Rand} x(x)$ where $x=1$.

4. Rand $x(x)$ : A host sends $x$ elephant flows to any other end host in the same topology with a uniform probability.
5. Randbij: A host sends one elephant flow to some other host according to a bijective mapping of all hosts. This is a special case of Random pattern which may be created by certain cluster computing applications.

\subsection{Small Topology Simulation Results}

In this set of simulations, 16 hosts (acting as clients) first establish TCP connections with some designated peers (acting as servers) according to the specified traffic pattern. After that, these clients begin to send elephant flows to their peers constantly. Each simulation lasts 60 seconds, and each host measures the incoming throughput during the whole process. We use the results for all hosts in the middle 40 seconds as the aggregate throughput.

Figure 6a shows the average aggregate throughput for a variety of Shift traffic patterns with different parameters. For Shift parameter $i=1$, all three methods have good performance. DiFS achieves the highest throughput for all $i$ values and outperforms ECMP significantly when $i$ is greater than 2. DiFS has a significant lead over Hedera and Dard when $i=9$ and 11 . Note a larger value of $i$ indicates less traffic locality. Hence DiFS is more robust than the other methods for traffic locality.

Figure $6 \mathrm{~b}$ shows the average aggregate throughput for Staggered patterns. Similar to the Shift results, DiFS has the highest throughput for most cases. In two cases (stag2(.2,.3) and stag3(.2,.3)), DiFS's throughput is marginally less than that of Hedera and Dard respectively. We might find that the absolute bandwidth values of all three methods in this set of simulations are less than those in the Shift simulations. According to our results on non-blocking switches and links (not shown in the figure), the average throughput for Staggered is also limited to 10-12 Gbps due to the hotspots created by the traffic pattern. DiFS results are relatively closer to the limit than the others.

Figure 6c depicts the throughput for Random patterns. For all cases except one, DiFS outperforms the 


\begin{tabular}{|l|c|c|c|c|}
\hline & ECMP & Hedera & Dard & DiFS \\
\hline \hline Shuffle time (s) & 249.82 & 204.87 & 210.83 & 179.48 \\
\hline $\begin{array}{l}\text { Aver. completion } \\
\text { time (s) }\end{array}$ & 224.78 & 178.53 & 191.25 & 157.20 \\
\hline $\begin{array}{l}\text { Aver. throughput } \\
\text { (Gbps) }\end{array}$ & 4.31 & 5.30 & 4.61 & 6.10 \\
\hline $\begin{array}{l}\text { Aver. out-of-order to } \\
\text { in-order ratio }\end{array}$ & 0.006 & 0.006 & 0.006 & 0.006 \\
\hline $\begin{array}{l}\text { Max. out-of-order to } \\
\text { in-order ratio }\end{array}$ & 0.643 & 0.750 & 0.750 & 0.4 \\
\hline $\begin{array}{l}\text { Aver. out-of-order } \\
\text { window size }\end{array}$ & 0.00 & 14.75 & 13.72 & 28.66 \\
\hline $\begin{array}{l}\text { Max. out-of-order } \\
\text { window size }\end{array}$ & 0.00 & 69.00 & 68.00 & 123.00 \\
\hline
\end{tabular}

Table 1: results of shuffle simulations

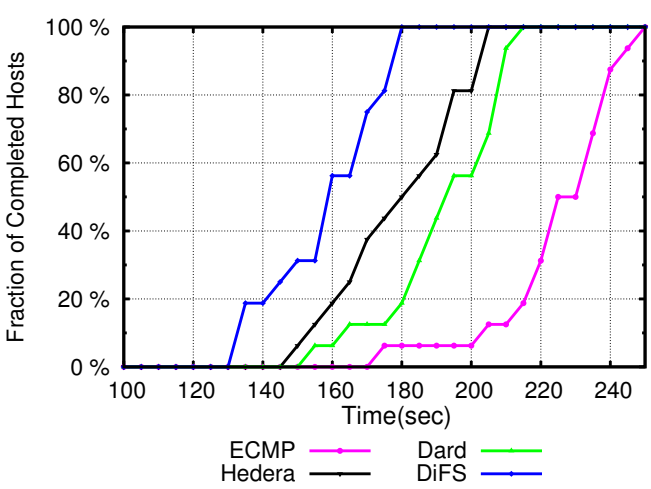

Figure 7: CDF of host completion time for data shuffle

other three protocols. In Random simulations, DiFS outperforms ECMP in the average throughput by at least $33 \%$ for most traffic patterns. For particular patterns, this value can be higher than $100 \%$. Compared to Hedera and Dard that uses global information, DiFS achieves higher throughput for the Randbij1 pattern and similar throughput for the others. We suspect there are two major reasons why Hedera achieves less bandwidth compared to DiFS: First, Hedera ignores intra-pod flows and degrades to ECMP when intra-pod flows are dominant. Second, Hedera with Simulated Annealing does not assign an explicit path for each flow. Instead, Hedera assigns a core switch for every single host, which may result in bottlenecks on the links connecting aggregate switches and edge switches.

\subsection{Dynamic Traffic: Data Shuffle}

We conduct simulations of all-to-all Data Shuffle in the 16-host multi-rooted tree topology to evaluate the performance of DiFS under dynamic traffic patterns.
Data Shuffle is an important operation for MapReducelike applications. Each host (acting as reducer) in the network will sequentially receive a large amount of data (500MB in our simulation) from all other hosts (acting as mapper) using TCP. Therefore, in total it is a $120 \mathrm{~GB}$ Data Shuffle. In order to avoid unnecessary hotspots, each host will access other hosts in a random order. We also assume there is no disk operation during the whole process. We measure the shuffle time, average completion time, and average throughput of the three methods. The shuffle time is the total time for the 120 GB Shuffle operation. The average completion time is the average value of the completion time of every host in the network. The average aggregate throughput refers to the sum of average throughput of every host.

We also measure two variables described in [5] during the Shuffle period in order to reflect the packet reordering problem. The first variable is the ratio of the number of packets delivered out-of-order to the number of packets provided in-order in TCP by the senders. The second variable is the out-of-order packet window size, defined as the average gap in the packet sequence numbers observed by the receivers.

Table 1 shows that our algorithm outperforms ECMP by $28 \%$, Hedera by around $13 \%$, and Dard by $15 \%$, in aggregate throughput. The most important metric is the shuffle time, i.e., the maximal completion time of all flows. The shuffle time of DiFS is less than $180 \mathrm{sec}-$ onds while the other methods use more than $200 \mathrm{sec}-$ onds. DiFS achieves the least shuffle time and average completion time per flow. In addition, DiFS causes less packet reordering compared to Hedera. ECMP has the least out-of-order packets because it is a static scheduling algorithm.

Figure 7 depicts the cumulative distribution function (CDF) of host completion time of the three methods. As observed from this figure, by the time DiFS finishes Shuffle operations, around $50 \%$ hosts of Hedera have completed their jobs and only 20\% hosts of Dard and $5 \%$ hosts of ECMP have finished their jobs. In general DiFS finishes flows much faster than all other protocols. All four methods have obvious variation in the completion time of different flows.

\subsection{Large Topology Simulation Results}

Figure 8 shows the aggregate throughput comparison using a 1024-host FatTree network $(k=16)$. We can find that ECMP performs worse in a large topology, compared with its performance in the 16-host network using the same traffic patterns. We suspect this is because the chances of collisions in path assignment for static hash functions increase when topology gets 


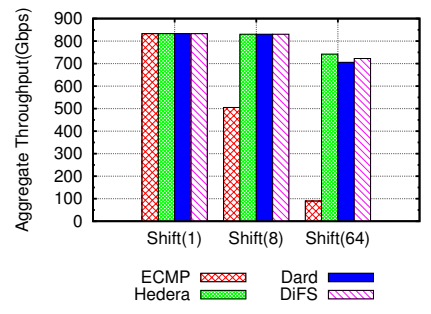

(a) Shift traffic pattern

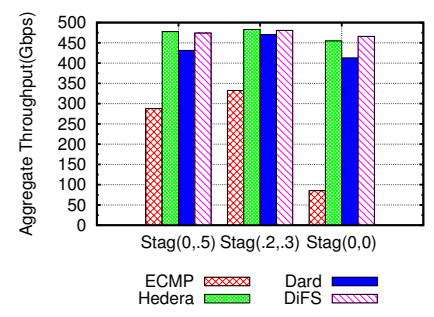

(b) Staggered traffic pattern

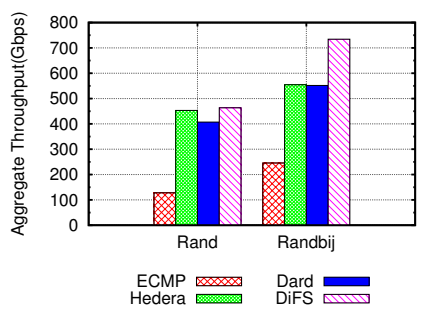

(c) Random traffic pattern

Figure 8: Aggregate throughput comparison for bulk analysis

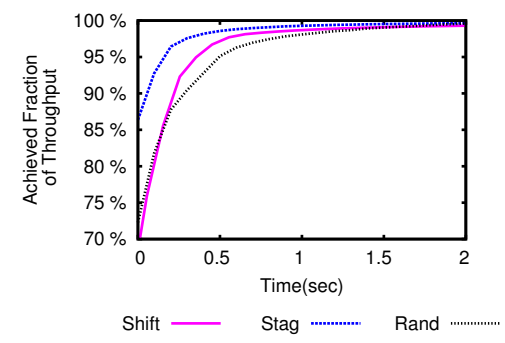

Figure 9: Convergence time of DiFS in the 1024-host network

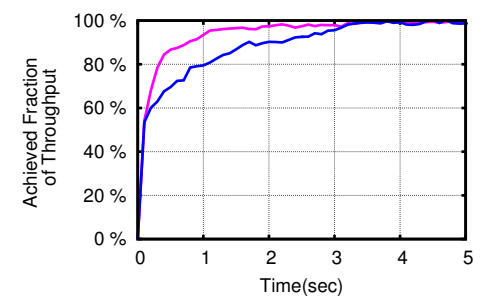

$10 \mathrm{~ms} \longrightarrow 100 \mathrm{~ms}$

Figure 10: Convergence time of DiFS with different control loops

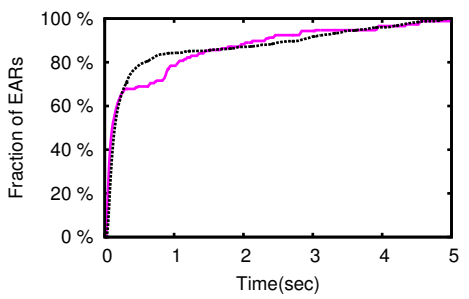

128 host -1024 host $\ldots . . . . . . . . . .$.

Figure 11: Cumulative distribution of EARreceiving times larger. We also noticed that the performance gap between Hedera and DiFS shrinks in the 1024-host network compared to that in the 16-host network due to the decreased portion of intra pod flows. However, DiFS still has the highest aggregate throughput in general except for two traffic patterns among the three figures.

\subsection{Convergence speed and control overhead}

\section{Convergence speed.}

Convergence speed is a critical performance metric for DiFS, because DiFS is a distributed solution rather than a centralized algorithm. We measure the convergence speed of DiFS for different traffic patterns using FatTree topologies. In Figure 9 we show the achieved fraction of throughput of DiFS versus time for different traffic patterns in the 1024-host network. Even with Random traffic our algorithm may still converge to a steady state within 5 seconds. We also compare the convergence speed against the frequency of control loops in 1024 host networks using Randbij patterns. Figure 10 compares the convergence speed of DiFS with $10 \mathrm{~ms}$ control loops to $100 \mathrm{~ms}$ control loops. Although smaller frequency yields longer converge time, the throughput still converge to relatively stable state in three seconds and achieves more than $80 \%$ throughput in the first second. We may conclude that our protocol is robust under different frequencies of control loops.

\begin{tabular}{|c|c|c|c|}
\hline $\mathrm{k}$ & Host & EAR & Control Overhead (KB) \\
\hline \hline 4 & 16 & 4 & 0 \\
\hline 8 & 128 & 304 & 7.72 \\
\hline 16 & 1024 & 4113 & 104.43 \\
\hline 32 & 8192 & 45183 & 1147.22 \\
\hline
\end{tabular}

Table 2: Control overhead of DiFS for random traffic patterns

\section{Control Overhead.}

As a distributed solution, the computation cost of DiFS is very low because switch only needs to consider its local flows. Hence, we mainly focus on the communication overhead of DiFS, which is measured by the number of EAR messages. Aside from communication overhead, too many EAR messages may cause performance degradation because flows may be requested to change their paths back and forth.

Table 2 shows the number of EARs sent by switches under random traffic patterns in FatTree networks with different sizes. In the measurement, we assume the size of each message is 26 Bytes, which includes the size of flow identifier and the address of recommended core or aggregate switch in an EAR. As shown in the table, for an 8192-host FatTree network, DiFS only generates control messages in a total size of around $1 \mathrm{MB}$. Figure 11 shows the CDF of EAR-receiving times. Within 


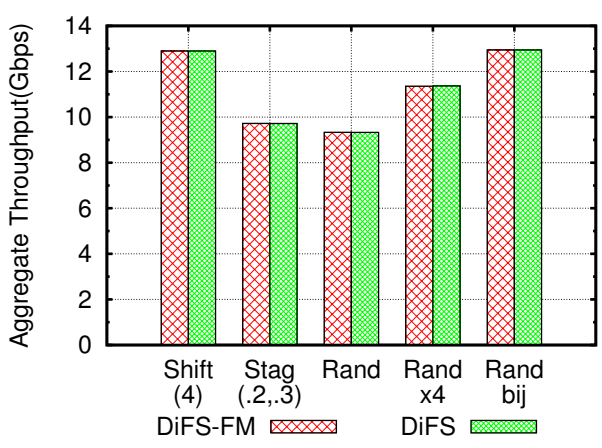

(a) 16-host network

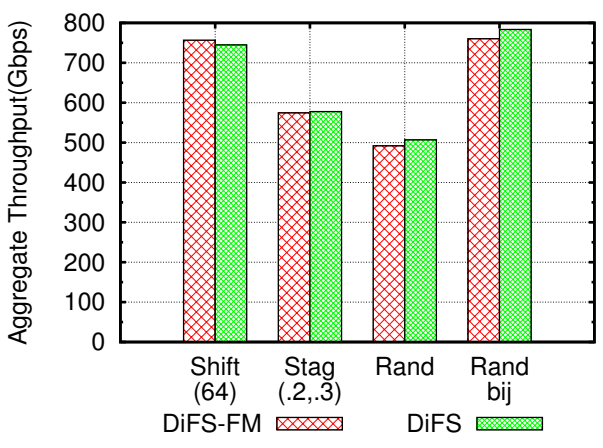

(b) 1024-host network

Figure 12: Flow bandwidth measurement vs flow counting

5 seconds, all EARs have sent and received, and around $80 \%$ EARs are received in the first second. We also measure the control overhead under dynamic traffic patterns. The average number of EAR messages is 15.4 for the experiments in Section 5.3.

\subsection{Failure recovery}

We consider three kinds of failures in simulation: single link failures, multiple independent link failures and single node failures. For each failure pattern, we consider three kinds of client transmission rate: $50 \mathrm{MB} / \mathrm{s}$, $65 \mathrm{MB} / \mathrm{s}$ and $80 \mathrm{MB} / \mathrm{s}$. The failures are generated randomly and will last for 10 seconds. We also recorded the throughput 5 seconds before and after the failure. Figure 13 shows the throughput for different sending rates under single link failures. When each sender is send data in $50 \mathrm{MB} / \mathrm{s}$, a single link is down at the 5th second and we can observe that aggregate bandwidth is dropped by around $15 \%$ without failure handling. However, the aggregate bandwidth does not drop so much if DiFS rerouted the impacted flows. Even though the aggregate bandwidth may drop when sending rate gets larger $(65 \mathrm{MB} / \mathrm{s}$ and $80 \mathrm{MB} / \mathrm{s})$, the overall bandwidth with failure handling is not worse than that without failure handling and no flow will be suspended due to network failure. Figure 14 and Figure 13 exhibit similar behaviour under independent link failures and single node failures.

\subsection{Flow count versus flow bandwidth consumption}

DiFS use the number of elephant flows as the metric for load balancing. Obviously, not all elephant flows have equal bandwidth consumptions, i.e., sending rates. As discussed in Section 3.2, DiFS cannot estimate the flow bandwidth consumption due to lack of global information. A substitution for bandwidth consumption estimation is to measure the sending rate of each flow on the current path. Unfortunately, a flow's current sending rate doest not reflect its maximum bandwidth consumption [8]. In the evaluation, the sending rate is controlled by TCP congestion control algorithms. Every host makes its best effort to send packets. We also implemented a variant of DiFS which uses measured flow sending rate as the metric for load balancing, denoted as DiFS-FM. We compare both algorithms in Figure 12a and Figure 12b. The results tell that DiFS-FM has similar performance compared to DiFS that uses flow count. Therefore, there is no need to deploy a particular module to keep measuring sending rates in switches.

\subsection{Summary of results}

To summarize the performance evaluation, we compare the important properties of adaptive switching methods in Table 3. Our results show that DiFS can achieve similar or even higher throughput than Hedera and Dard that require network-wide information for routing decisions. As a local, switch-only solution, DiFS does not have the limitations of central and hostbased methods such as bottleneck of a single controller and massive monitoring messages. Compared to the state-of-art networking techniques, DiFS only requires either the SDN support or simple special switch logic.

\section{Related Works}

Recently there have been a great number of proposals for data center network topologies that provide high bisection bandwidth [4, 2, 30, 31, 32]. However, current routing protocols like ECMP [6] usually suffer from elephant flow collisions and bandwidth loss. Application layer scheduling like Orchestra [33] usually focuses on 
Table 3: Important properties of adaptive switching methods.

\begin{tabular}{c||cccc}
\hline & ECMP [6] & Hedera [8] & Dard [3] & DiFS (this work) \\
\hline Network throughput & Benchmark & Higher than ECMP & High than ECMP & $\approx$ Hedera and Dard \\
Flow completion & Benchmark & faster than ECMP and Dard & faster than ECMP & faster than other three \\
Decision making & Local info. & Network-wide info. & Network-wide info. & Local info. \\
Scalability problem? & Scalable & Bottleneck of a single controller & Massive monitoring msgs & Scalable \\
Compatibility & Standard & SDN support \& monitoring tools & Changes on hosts & SDN support or switch logic \\
\hline
\end{tabular}

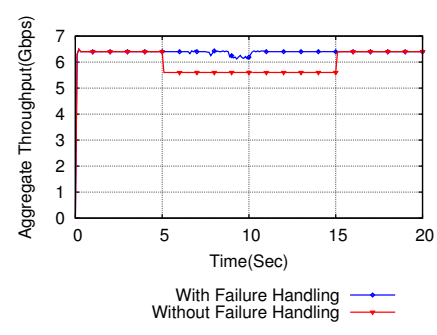

(a) $50 \mathrm{MBps}$

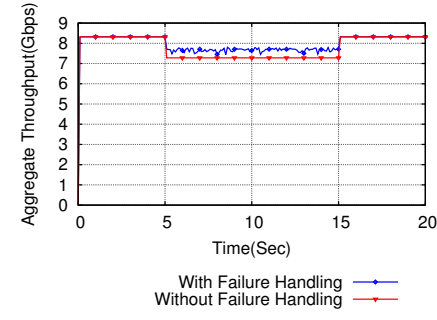

(b) $65 \mathrm{MBps}$

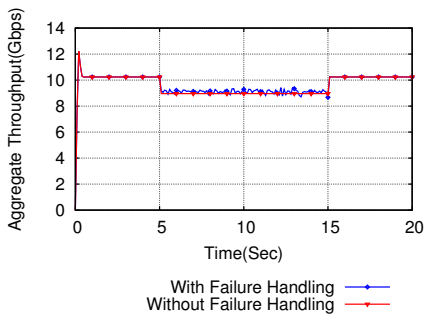

(c) $80 \mathrm{MBps}$

Figure 13: Aggregate throughput comparison for single link failure

higher level scheduling policies such as transfer prioritizing and ignores multipathing issues in data center networks. Transport layer solutions like DCTCP [13] and MPTCP [14] optimize the resource share on fixed paths among flows. This work focuses on adaptive switching solutions.

Centralized flow routing $[8,34]$ usually relies on a central controller and schedules flow path at every control interval. Aside from the additional hardware and software support for communication and computation, centralized solutions may be hard to scale out due to the single point of the controller. Recent research [10, 11] shows that centralized solutions must employ parallelism and fast route computation heuristics to support observed traffic patterns.

Host-based solutions [3] enable end hosts select flow path simultaneously to enhance parallelism. Dard [3] allows each host to select flow path based on network conditions. However, Dard has potential scalability issues due to massive monitoring messages to every host. Besides, deployment of host-based solutions requires updates on legacy systems and applications.

Switch-only protocols $[35,9,5,36]$ are also proposed. However most of them require flow splitting which may cause significant packet reordering. TeXCP [35], as an online distributed Traffic Engineering protocols, performs packet-level load balancing by using splitting schemes like FLARE [37]. Localflow [9] refines a naive link balancing solution and minimizes the number of flows that are split. Dixit et al. [36] uses random packet spraying to split flows to multiple paths to minimize the hurts to TCP. DiFS does not split a flow in order to avoid packet reordering.

\section{Conclusion}

This paper proposes DiFS, a local, lightweight, and switch-only protocol for adaptive packet switching in data center networks. Switches running DiFS cooperate to achieve flow-to-link balance by avoiding both local and remote collisions. Experimental results show that our algorithm can outperform the well-known distributed solution ECMP, a centralized scheduling algorithm Hedera, and a host-based protocol Dard. We will investigate flow scheduling for general network topologies in future work.

\section{Acknowledgments}

Ye Yu and Chen Qian were supported by University of Kentucky College of Engineering Faculty Startup Grant and National Science Foundation grant CNS1464335 .

\section{References}

[1] M. Al-Fares, A. Loukissas, A. Vahdat, A scalable, commodity data center network architecture, in: Proc. of ACM SIGCOMM, 2008.

[2] R. N. Mysore, et al., Portland: a scalable fault-tolerant layer 2 data center network fabric, in: Proceedings of ACM SIGCOMM, 2009.

[3] X. Wu, X. Yang, Dard: Distributed adaptive routing for datacenter networks, in: Proceedings of IEEE ICDCS, 2012. 


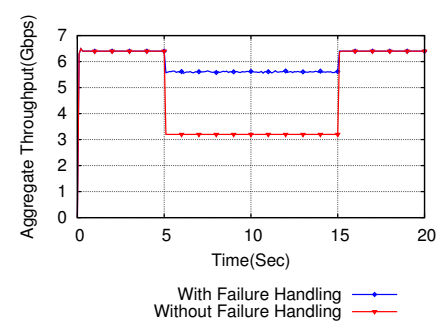

(a) 50MBps

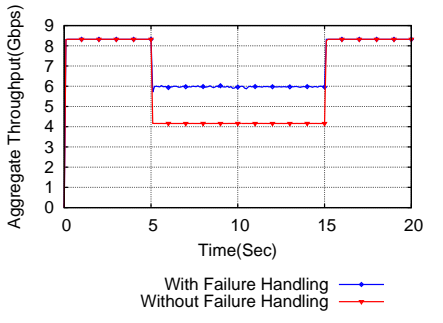

(b) $65 \mathrm{MBps}$

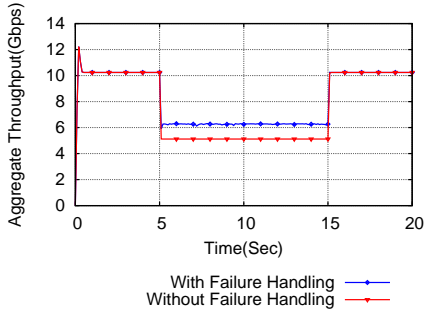

(c) $80 \mathrm{MBps}$

Figure 14: Aggregate throughput comparison for independent link failure

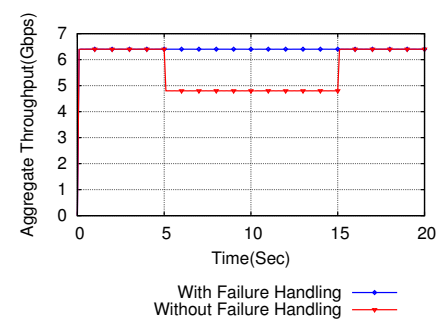

(a) $50 \mathrm{MBps}$

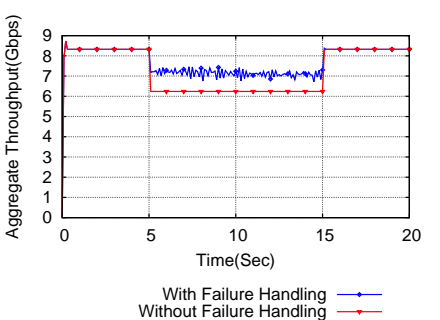

(b) $65 \mathrm{MBps}$

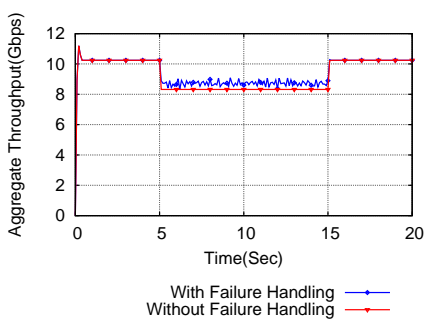

(c) $80 \mathrm{MBps}$

Figure 15: Aggregate throughput comparison for single node failure

[4] A. Greenberg, J. R. Hamilton, N. Jain, S. Kandula, C. Kim, P. Lahiri, D. A. Maltz, P. Patel, S. Sengupta, V12: a scalable and flexible data center network, in: Proceedings of ACM SIGCOMM, 2009.

[5] E. Zahavi, I. Keslassy, A. Kolodny, Distributed adaptive routing for big-data applications running on data center networks, in: Proceedings of ACM/IEEE ANCS, 2012.

[6] C. Hopps, Analysis of an equal-cost multi-path algorithm, RFC 2992.

[7] N. McKeown, T. Anderson, H. Balakrishnan, G. Parulkar, L. Peterson, J. Rexford, S. Shenker, J. Turner, Openflow: Enabling innovation in campus networks, SIGCOMM Comput. Commun. Rev.

[8] M. Al-Fares, S. Radhakrishnan, B. Raghavan, N. Huang, A. Vahdat, Hedera: dynamic flow scheduling for data center networks, in: Proceedings of USENIX NSDI, 2010.

[9] S. Sen, D. Shue, S. Ihm, M. J. Freedman, Scalable, opitmal flow routing in datacenters via local link balancing, in: Proceedings of ACM CoNEXT, 2013.

[10] S. Kandula, S. Sengupta, A. Greenberg, P. Patel, R. Chaiken, The nature of data center traffic: measurements \& analysis, in: Proceedings of ACM IMC, 2009.

[11] T. Benson, A. Akella, D. A. Maltz, Network traffic characteristics of data centers in the wild, in: Proceedings of ACM IMC, 2010.

[12] J. Perry, A. Ousterhout, H. Balakrishnan, D. Shah, H. Fugal, Fastpass: A centralized zero-queue datacenter network, in: Proceedings of the 2014 ACM conference on SIGCOMM, ACM, 2014, pp. 307-318.

[13] M. Alizadeh, A. Greenberg, D. A. Maltz, J. Padhye, P. Patel, B. Prabhakar, S. Sengupta, M. Sridharan, Dctcp: Efficient packet transport for the commoditized data center, in: Proceedings of ACM SIGCOMM, 2010.

[14] D. Wischik, C. Raiciu, A. Greenhalgh, M. Handley, Design, implementation and evaluation of congestion control for multipath tcp, in: Proceedings of USENIX NSDI, 2011.

[15] A. Dixit, P. Prakash, R. R. Kompella, On the efficacy of finegrained traffic splitting protocols in data center networks, in: Proceedings of ACM SIGCOMM, 2011.

[16] K. C. Leung, V. Li, D. Yang, An overview of packet reordering in transmission control protocol (tcp): Problems, solutions, and challenges, IEEE Transactions on Parallel and Distributed Systems.

[17] X. Yang, D. Clark, A. Berger, Nira: A new inter-domain routing architecture, IEEE/ACM Transactions on Networking.

[18] Z. Ding, R. R. Hoare, A. K. Jones, R. Melhem, Level-wise scheduling algorithm for fat tree interconnection networks, in: Proceedings of the 2006 ACM/IEEE conference on Supercomputing, ACM, 2006, p. 96.

[19] Catalyst switch architecture and operation, http://www.cisco.com/networkers/nw03/presos/docs/RST2011.pdf.

[20] Hpe flexnetwork switch chassis, http://www8.hp.com/us/en/products/networking-switches/.

[21] S. Han, K. Jang, A. Panda, S. Palkar, D. Han, S. Ratnasamy, Softnic: A software nic to augment hardware, Tech. Rep. UCB/EECS-2015-155, EECS Department, University of California, Berkeley (May 2015).

URL http://www.eecs.berkeley.edu/Pubs/TechRpts/ 2015/EECS-2015-155.html

[22] D. Zhou, B. Fan, H. Lim, M. Kaminsky, D. G. Andersen, Scalable, high performance ethernet forwarding with cuckooswitch, in: Proceedings of the Ninth ACM Conference on Emerging Networking Experiments and Technologies, CoNEXT '13, ACM, New York, NY, USA, 2013, pp. 97-108. doi:10.1145/2535372.2535379.

URL http: //doi . acm.org/10.1145/2535372. 2535379

[23] Intel data plane development kit, http://dpdk.org/.

[24] M. Yu, L. Jose, R. Miao, Software defined traffic measurement with opensketch, in: Proc. of USENIX NSDI, 2013. 
[25] H. Ballani, P. Costa, T. Karagiannis, A. Rowstron, Towards predictable datacenter networks., in: Proc. of SIGCOMM, 2011.

[26] P. Gill, N. Jain, N. Nagappan, Understanding network failures in data centers: Measurement, analysis, and implications, in: Proc. of ACM SIGCOMM, 2011.

[27] F. P. Tso, D. Pezaros, Baatdaat: Measurement-based flow scheduling for cloud data centers, in: Proc. of ISCC, 2013.

[28] A. Singla, C.-Y. Hong, L. Popa, P. B. Godfrey, Jellyfish: Networking data centers randomly, in: Proc. of USENIX NSDI 2012.

[29] J. Dean, S. Ghemawat, Mapreduce: simplified data processing on large clusters, Communications of the ACM.

[30] A. Greenberg, P. Lahiri, D. A. Maltz, P. Patel, S. Sengupta, Towards a next generation data center architecture: Scalability and commoditization, in: Proc. of ACM PRESTO, 2008.

[31] C. Guo, et al., Bcube: a high performance, server-centric network architecture for modular data centers, in: Proc. of ACM SIGCOMM, 2009.

[32] C. Guo, et al., Dcell: a scalable and fault-tolerant network structure for data centers, in: Proc. of ACM SIGCOMM, 2008.

[33] M. Chowdhury, M. Zaharia, J. Ma, M. I. Jordan, I. Stoica, Managing data transfers in computer clusters with orchestra, in: Proc. of ACM SIGCOMM, 2011.

[34] T. Benson, A. Anand, A. Akella, M. Zhang, Microte: fine grained traffic engineering for data centers, in: Proc. of ACM CoNEXT, 2011.

[35] S. Kandula, D. Katabi, B. Davie, A. Charny, Walking the tightrope: responsive yet stable traffic engineering, in: Proceedings of ACM SIGCOMM, 2005

[36] A. Dixit, P. Prakash, Y. C. Hu, R. R. Kompella, On the impact of packet spraying in data center networks, in: Proc. of IEEE INFOCOM, 2013.

[37] S. Sinha, S. Kandula, D. Katabi, Harnessing tcps burstiness using flowlet switching, in: Proc. of ACM HotNets, 2004.

Wenzhi Cui is a Ph.D. student at the Department of Computer Science, University of Texas at Austin. He received the B.Sc. degree from Nanjing University in Software Engineering. His research interests including computer networking and distributed systems.

Ye Yu is a Ph.D. student at the Department of Computer Science, University of Kentucky. He received the B.Sc. degree from Beihang University. His research interests including data center networks and software defined networking.

Chen Qian is an Assistant Professor at the Department of Computer Science, University of Kentucky. He received the B.Sc. degree from Nanjing University in 2006, the M.Phil. degree from the Hong Kong University of Science and Technology in 2008, and the Ph.D. degree from the University of Texas at Austin in 2013, all in Computer Science. His research interests include computer networking, data-center networks and cloud computing, and Internet of Things. He has published more than 30 research papers in a number of top conferences and journals. He has served in the organizing committees and technical program committees of a number of conferences, including the TPC co-chair of ACM CSAR (with SenSys'16), TPC track chair of ICCCN'16, TPC co-chair of ICNP student workshop 2013. He is an editor of the Cyber-Physical Systems Journal. He received the Best Paper Award of ACM MSCC 2015. 